Article

\title{
Pulmonary Arteriovenous Pressure Gradient and Time-Averaged Mean Velocity of Small Pulmonary Arteries Can Serve as Sensitive Biomarkers in the Diagnosis of Pulmonary Arterial Hypertension: A Preclinical Study by 4D-Flow MRI
}

\author{
Ali Nahardani ${ }^{1,2}{ }^{2}$, Simon Leistikow ${ }^{3}$, Katja Grün ${ }^{4}$, Martin Krämer ${ }^{1}{ }^{\mathbb{D}}$, Karl-Heinz Herrmann ${ }^{1}$, \\ Andrea Schrepper ${ }^{5}$, Christian Jung ${ }^{6} \mathbb{D}$, Sara Moradi ${ }^{2}$, Paul Christian Schulze ${ }^{4}$, Lars Linsen ${ }^{3}$, \\ Jürgen R. Reichenbach ${ }^{1}$, Verena Hoerr ${ }^{1,2,7, *,+}$ and Marcus Franz ${ }^{4,+}$ (D)
}

1 Medical Physics Group, Institute of Diagnostic and Interventional Radiology, Jena University Hospital, Friedrich Schiller University Jena, 07747 Jena, Germany; nahardani.ali@gmail.com (A.N.); Martin.Kraemer@med.uni-jena.de (M.K.); Karl-Heinz.Herrmann@med.uni-jena.de (K.-H.H.); Juergen.Reichenbach@med.uni-jena.de (J.R.R.)

2 Heart Center Bonn, Department of Internal Medicine II, University Hospital Bonn, 53127 Bonn, Germany; sara.moradi.567@gmail.com

3 Department of Mathematics and Computer Science, Institute of Computer Science, Westfälische Wilhelms-Universität Münster, 48149 Munster, Germany; simon.leistikow@uni-muenster.de (S.L.); linsen@uni-muenster.de (L.L.)

check for updates

Citation: Nahardani, A.; Leistikow, S.; Grün, K.; Krämer, M.; Herrmann K.-H.; Schrepper, A.; Jung, C.; Moradi, S.; Schulze, P.C.; Linsen, L.; et al. Pulmonary Arteriovenous

\section{Pressure Gradient and}

Time-Averaged Mean Velocity of Small Pulmonary Arteries Can Serve as Sensitive Biomarkers in the Diagnosis of Pulmonary Arterial Hypertension: A Preclinical Study by 4D-Flow MRI. Diagnostics 2022, 12, 58. https://doi.org/10.3390/ diagnostics12010058

Academic Editor: Andrea D. Annoni

Received: 15 November 2021

Accepted: 21 December 2021

Published: 28 December 2021

Publisher's Note: MDPI stays neutral with regard to jurisdictional claims in published maps and institutional affiliations.

Copyright: (C) 2021 by the authors. Licensee MDPI, Basel, Switzerland. This article is an open access article distributed under the terms and conditions of the Creative Commons Attribution (CC BY) license (https:// creativecommons.org/licenses/by/ $4.0 /)$.
4 Department of Internal Medicine I, Division of Cardiology, Angiology, Pneumology, and Intensive Medical Care, Jena University Hospital, 07747 Jena, Germany; Katja.Gruen@med.uni-jena.de (K.G.); Christian.Schulze@med.uni-jena.de (P.C.S.); Marcus.Franz@med.uni-jena.de (M.F.)

5 Department of Cardiothoracic Surgery, Jena University Hospital, 07747 Jena, Germany; Andrea.Schrepper@med.uni-jena.de

6 Department of Internal Medicine, Division of Cardiology, University Hospital Düsseldorf, 40225 Dusseldorf, Germany; Christian.Jung@med.uni-duesseldorf.de

7 Translational Research Imaging Center (TRIC), Clinic for Radiology, University Hospital Münster, 48149 Munster, Germany

* Correspondence: vhoerr@uni-muenster.de

+ These authors contributed equally to this work.

Abstract: (1) Background: Pulmonary arterial hypertension (PAH) is a serious condition that is associated with many cardiopulmonary diseases. Invasive right heart catheterization (RHC) is currently the only method for the definitive diagnosis and follow-up of PAH. In this study, we sought a non-invasive hemodynamic biomarker for the diagnosis of PAH. (2) Methods: We applied prospectively respiratory and cardiac gated $4 \mathrm{D}$-flow MRI at a $9.4 \mathrm{~T}$ preclinical scanner on three different groups of Sprague Dawley rats: baseline $(n=11)$, moderate PAH $(n=8)$, and severe PAH $(n=8)$. The pressure gradients as well as the velocity values were analyzed from $4 \mathrm{D}$-flow data and correlated with lung histology. (3) Results: The pressure gradient between the pulmonary artery and vein on the unilateral side as well as the time-averaged mean velocity values of the small pulmonary arteries were capable of distinguishing not only between baseline and severe PAH, but also between the moderate and severe stages of the disease. (4) Conclusions: The current preclinical study suggests the pulmonary arteriovenous pressure gradient and the time-averaged mean velocity as potential biomarkers to diagnose PAH.

Keywords: pulmonary hypertension; cardiac magnetic resonance; treatment response; hemodynamics; 4D-flow

\section{Introduction}

Pulmonary hypertension $(\mathrm{PH})$ is a life-threatening condition that is defined as an increase in mean pulmonary artery pressure above $20 \mathrm{mmHg}$ when measured by right 
heart catheterization (RHC) at rest [1,2]. Clinically, pulmonary arterial hypertension (PAH) is categorized as the first group in the PH classification, which is described in detail in the clinical guidelines of the European Society of Cardiology [1]. The clinical manifestations of PAH are often nonspecific and usually arise from right ventricular dysfunction in advanced stages $[1,3]$. Routine blood gas and respiratory function tests, electrocardiography (ECG), chest X-ray, as well as blood and serological tests can produce different results for a variety of conditions and indicate a number of possible underlying diseases [1,4]. Therefore, further evaluations by means of additional diagnostic tools are recommended for differential diagnosis. RHC is the diagnostic standard for the definite diagnosis of $\mathrm{PH}$, the assessment of the severity of hemodynamic impairment in its course and for further follow-ups [1,5-7]. However, there are a variety of pitfalls when assessing and interpreting the parameters determined by RHC; for example, the measurement of the pulmonary artery wedge pressure at an over- or under-wedge position can lead to incorrect results [8]. In addition, as an invasive diagnostic tool, RHC can cause intra- or postprocedure complications, such as hematomas, pulmonary artery ruptures, or damage to the electrical conduction system of the heart [9]. Furthermore, it is contraindicated under certain circumstances such as in mechanical tricuspid or pulmonary valve replacement, right heart masses, etc. [7]. In contrast to RHC, transthoracic echocardiography (TTE) is a non-invasive method that cannot directly measure pulmonary vascular resistance or any other related indices to vascular pressure, but plays an important role in assessing the likelihood of $\mathrm{PAH}$, the development of right heart failure, or the necessity for catheterization in symptomatic patients $[1,10]$. Nuclear imaging and computed tomography techniques are also indicated in pulmonary hypertension assessments [11-13], but suffer from high effective dose exposures. Cardiac magnetic resonance (CMR) can improve patient care in clinics substantially [14] as it is the gold standard for quantifying right-heart function $[15,16]$ and is useful to PH evaluations $[5,15,17]$. In recent years, the development of $4 \mathrm{D}$-flow CMR has opened a new direction for the qualitative and quantitative assessment of cardiovascular hemodynamics and function [18-20]. In previous studies, various CMR hemodynamic indices (such as peak velocity, blood flow, etc.) were compared to RHC $[19,21,22]$. However, none of the evaluated biomarkers correlated very strongly with the catheterization results. Among all the parameters, the most promising non-invasive hemodynamic index was the time-averaged mean velocity of the main pulmonary artery [19]. For this purpose, we evaluated this index in the small pulmonary arteries in 4D-flow and its correlation with histology to assess its suitability for diagnosing PAH. In addition, we calculated the pulmonary arteriovenous pressure gradient (i.e., the pressure gradient between the pulmonary small arteries and veins of the unilateral side) in all the experimental groups to evaluate its diagnostic utility for PAH. To confirm the influence of the experimental PAH model on heart mechanics, we calculated tricuspid annular plane systolic excursion (TAPSE) by means of TTE and right-ventricular ejection fraction (RVEF) by using MRI. Finally, a comprehensive correlation analysis of all the CMR findings in relation to the histology results was performed.

\section{Materials and Methods}

- Animal model: A total of 27 male Sprague Dawley rats (Charles River Laboratories, Sulzfeld, Germany) with an average weight of $330 \pm 39.9 \mathrm{~g}$ were included in the study and divided into three different experimental groups: (A) Baseline $(n=11$, with a nuchal subcutaneous injection of $300 \mu \mathrm{L} \mathrm{NaCl} \mathrm{0.9 \% );} \mathrm{(B)} \mathrm{Severe} \mathrm{PAH}(n=8$, with a single-dose subcutaneous injection of $60 \mathrm{mg} / \mathrm{kg}$ monocrotaline-Carl Roth, Karlsruhe, Germany-dissolved in $300 \mu \mathrm{L} \mathrm{NaCl} 0.9 \%$ ); and (C) Moderate PAH ( $n=8$, with the same medication as the severe PAH group and an additional oral application of $15 \mathrm{mg} / \mathrm{kg}$ macitentan-Actelion Pharmaceuticals Ltd., Allschwil, Switzerland-from day 14 to 28). All invasive and non-invasive diagnostic procedures were performed 4 weeks after disease induction. The general anesthesia protocol was chosen depending on the diagnostic procedure: all the non-invasive procedures were conducted 
under inhalation of isoflurane $\sim 3 \%$ (due to the long tubing between the vaporizers and the MRI system) to keep the animals' respiration in the range of 30 to 50 per minute. Perfusion was carried out under deep anesthesia with the intra-peritoneal administration of a single dose of $100 \mathrm{mg} / \mathrm{kg}$ body weight of ketamine (volume $450 \mu \mathrm{L}$ ) and $10 \mathrm{mg} / \mathrm{kg}$ body weight of xylazine (volume $250 \mu \mathrm{L}$ ). All the experiments were conducted in accordance with approved ethical guidelines (see the section on the Institutional Review Board Statement).

- Cardiac Magnetic Resonance: Prospectively, a cardiac and respiratory triggered 4Dflow stack-of-stars phase-contrast sequence was performed on a 9.4 T BioSpec USR 94/20 imaging scanner with ParaVision 6.0.1 software (Bruker, Ettlingen, Germany). All the animals were examined by a vendor-supplied $72 \mathrm{~mm}$-diameter quadrature volume resonator with the following sequence parameters. Flow encoding scheme: HADAMARD; TR = $10 \mathrm{~ms} ; \mathrm{TE}=1.1 \mathrm{~ms} ; \mathrm{FA}=10^{\circ} ; \mathrm{BW}=100 \mathrm{kHz}$; under-sampling factor $=1.4-1.6$; averages $=1$; resolution $=(375 \times 375 \times 375) \mu \mathrm{m}^{3} ; \mathrm{VENC}=75-200 \mathrm{~cm} / \mathrm{s}$. The data were reconstructed offline using regridding with iterative sampling density estimation. All the functional and hemodynamic parameters were quantified using manual region-of-interests (ROI). The time-averaged mean-velocities were calculated in the large (main, right and left pulmonary arteries) and small pulmonary arteries. Furthermore, the pulmonary arteriovenous pressure gradients were calculated through the use of modified Bernoulli's equation from 4D-flow data (Figure 1). All the parameters investigated by MRI are listed in Tables 1 and 2.

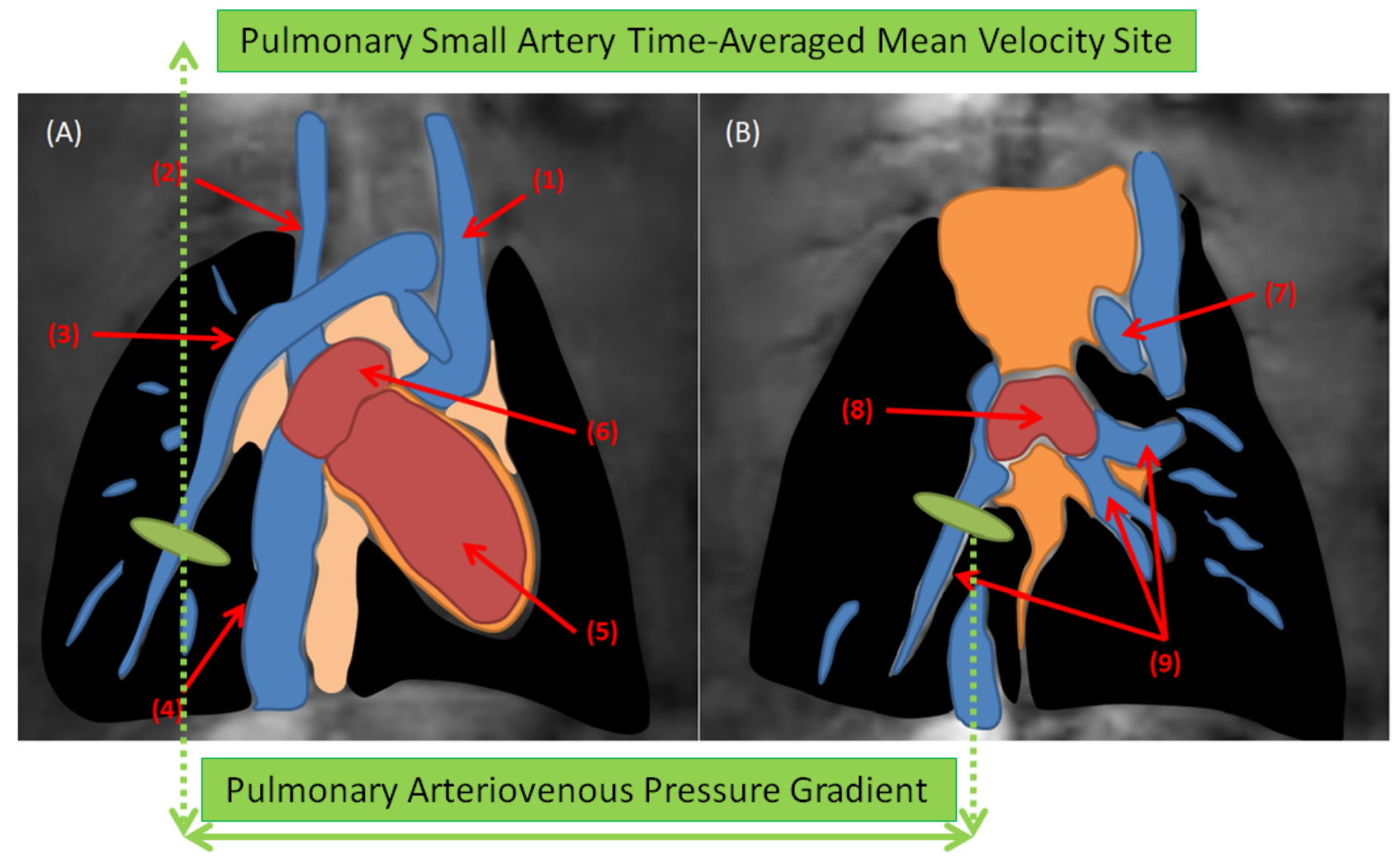

Figure 1. A schematic representation showing the region of interests (ROI) for the calculation of pulmonary arteriovenous pressure gradients and pulmonary small arteries' time-averaged mean velocities. The green label in (A) shows ROI on a small pulmonary artery, and in (B) depicts ROI on the ipsilateral pulmonary vein (Annotations: (1) left superior vena cava (2) right superior vena cava (3) right pulmonary artery along its small branch (4) inferior vena cava (5) right ventricle (6) right atrium (7) aorta (8) left atrium (9) pulmonary veins). 
Table 1. Results for CMR-derived hemodynamic parameters in the three experimental groups.

\begin{tabular}{|c|c|c|c|c|c|c|}
\hline \multirow{2}{*}{ MRI-Derived Index } & \multirow{2}{*}{ Abb. } & \multirow{2}{*}{ Unit } & \multirow{2}{*}{$\begin{array}{c}\text { Sig. } \\
(p<0.05)\end{array}$} & \multicolumn{3}{|c|}{ Group (Mean \pm Standard Deviation) } \\
\hline & & & & Baseline & Severe & Moderate \\
\hline Main pulmonary artery averaged velocity & $\mathrm{MPA}_{V \text {-mean }}$ & $\mathrm{cm} \cdot \mathrm{s}^{-1}$ & 1 & $30.43 \pm 7.90$ & $12.18 \pm 4.32$ & $22.28 \pm 11.41$ \\
\hline Right pulmonary artery averaged velocity & $\mathrm{RPA}_{\mathrm{V} \text {-mean }}$ & $\mathrm{cm} \cdot \mathrm{s}^{-1}$ & 1 & $23.15 \pm 4.97$ & $13.78 \pm 7.24$ & $19.15 \pm 10.78$ \\
\hline Left pulmonary artery averaged velocity & LPA $_{V-m e a n}$ & $\mathrm{~cm} \cdot \mathrm{s}^{-1}$ & 1 & $22.65 \pm 6.94$ & $12.77 \pm 2.23$ & $20.44 \pm 8.11$ \\
\hline Right pulmonary small artery averaged velocity & RPSA $_{V-\text { mean }}$ & $\mathrm{cm} \cdot \mathrm{s}^{-1}$ & 1 & $18.40 \pm 3.72$ & $7.43 \pm 3.40$ & $14.36 \pm 7.62$ \\
\hline Left pulmonary small artery averaged velocity & LPSA $_{V \text {-mean }}$ & $\mathrm{cm} \cdot \mathrm{s}^{-1}$ & 1,3 & $22.57 \pm 4.66$ & $7.47 \pm 4.07$ & $16.16 \pm 8.50$ \\
\hline Right pulmonary arteriovenous averaged pressure & $\mathrm{RPSA} \mathrm{RPV}_{\Delta \mathrm{P}-\text { mean }}$ & $\mathrm{mmHg}$ & 1,3 & $0.12 \pm 0.06$ & $0.018 \pm 0.02$ & $0.093 \pm 0.095$ \\
\hline $\begin{array}{l}\text { Left pulmonary arteriovenous averaged } \\
\text { pressure gradient }\end{array}$ & LPSA-LPV $_{\Delta \mathrm{P}-\text { mean }}$ & $\mathrm{mmHg}$ & 1,3 & $0.19 \pm 0.09$ & $0.02 \pm 0.02$ & $0.11 \pm 0.14$ \\
\hline
\end{tabular}

1, Two independent Mann-Whitney U tests between baseline and severe groups were significant $(p<0.05)$. 2, Two independent Mann-Whitney $U$ tests between baseline and moderate groups was significant $(p<0.05)$. 3, Two independent Mann-Whitney $U$ tests between severe and moderate groups were significant $(p<0.05)$.

Table 2. Results for different right heart functional indices derived from CMR and TTE in the three experimental groups.

\begin{tabular}{|c|c|c|c|c|c|c|}
\hline \multirow{2}{*}{ Functional Index } & \multirow{2}{*}{ Abb. } & \multirow{2}{*}{ Unit } & \multirow{2}{*}{$\begin{array}{c}\text { Sig. } \\
(p<0.05)\end{array}$} & \multicolumn{3}{|c|}{ Group (Mean \pm Standard Deviation) } \\
\hline & & & & Baseline & Severe & Moderate \\
\hline \multirow{2}{*}{$\begin{array}{l}\text { CMR: Right ventricle ejection fraction } \\
\text { TTE: Tricuspid annular plane } \\
\text { systolic excursion }\end{array}$} & RVEF & $\%$ & $1,2,3$ & $56.27 \pm 3.67$ & $17.97 \pm 2.31$ & $38.75 \pm 10.42$ \\
\hline & TAPSE & $\mathrm{mm}$ & 1,2 & $2.73 \pm 0.64$ & $1.03 \pm 0.17$ & $1.27 \pm 0.45$ \\
\hline
\end{tabular}

1, Two independent Mann-Whitney $U$ tests between baseline and severe groups were significant $(p<0.05)$. 2, Two independent Mann-Whitney U tests between baseline and moderate groups were significant $(p<0.05)$. 3, Two independent Mann-Whitney $U$ tests between severe and moderate groups were significant $(p<0.05)$.

- Transthoracic echocardiography: TTE was performed in all animals before CMR with a high-resolution ultrasound imaging system Vevo-770 (Visual Sonics, Toronto, ON, Canada) using a rodent specific $17 \mathrm{MHz}$ probe. The recorded TTE movies were analyzed by an experienced cardiologist and the calculated parameter is listed in Table 2.

- Histological assessment of lungs: The formalin-fixed, paraffin-embedded, and H\&Estained lung tissue sections were evaluated by two experienced scientists. PAHassociated tissue damage of the lungs was evaluated semi-quantitatively using an established and validated sum-score system of our group [23,24]. The list of the evaluated histological indices is summarized in Table 3. According to the scoring system, atelectasis area (AA), emphysema area (EA), peribronchial artery media hypertrophy (PAMH), peribronchial artery perivascular cellular edema (PAPCE), and small artery media hypertrophy (SAMH) were evaluated and scored individually. Subsequently, all the scores were added up and summarized by an index called "Lung assessment sum-score", or LASS, reflecting the overall pulmonary tissue changes due to PAH. 
Table 3. Results for pulmonary histological indices in the three experimental groups.

\begin{tabular}{|c|c|c|c|c|c|}
\hline \multirow{2}{*}{ Pulmonary Histopathology Index } & \multirow{2}{*}{ Abb. } & \multirow{2}{*}{ Sig. $(p<0.05)$} & \multicolumn{3}{|c|}{ Group (Mean \pm Standard Deviation) } \\
\hline & & & Baseline & Severe & Moderate \\
\hline Atelectasis area & AA & 1,3 & $0.80 \pm 0.44$ & $1.67 \pm 0.51$ & $1.00 \pm 0.00$ \\
\hline Emphysema area & EA & 2 & $0.20 \pm 0.447$ & $0.67 \pm 0.51$ & $1.25 \pm 0.50$ \\
\hline Peribronchial artery media hypertrophy & PAMH & $1,2,3$ & $0.40 \pm 0.54$ & $2.83 \pm 0.408$ & $2.00 \pm 0.00$ \\
\hline $\begin{array}{l}\text { Peribronchial artery perivascular } \\
\text { cellular edema }\end{array}$ & PAPCE & 1 & $0.00 \pm 0.00$ & $1.67 \pm 0.816$ & $1.00 \pm 1.15$ \\
\hline Small artery media hypertrophy & SAMH & $1,2,3$ & $0.00 \pm 0.00$ & $2.83 \pm 0.40$ & $2.00 \pm 0.00$ \\
\hline Lung assessment sum score & LASS & $1,2,3$ & $1.40 \pm 1.14$ & $9.67 \pm 0.81$ & $7.25 \pm 1.50$ \\
\hline
\end{tabular}

1, Two independent Mann-Whitney $U$ tests between baseline and severe groups were significant $(p<0.05)$. 2, Two independent Mann-Whitney U tests between baseline and moderate groups were significant $(p<0.05)$. 3, Two independent Mann-Whitney $U$ tests between severe and moderate groups were significant $(p<0.05)$.

- Statistical Analysis: The pairwise Mann-Whitney U test was performed to compare the means of various variables among the experimental groups statistically with a $p$-value significance threshold of $5 \%$. In addition, the Spearman correlation (between the scalar and ordinal variables) and Pearson correlation (between two scalar variables) tests were used for correlation analysis between two different parameters (Table 4). The strength of the correlation coefficients was classified according to the following scale: very strong correlation $(r=0.90$ to 1.0$)$, strong correlation $(r=0.70$ to 0.90$)$, moderate correlation $(\mathrm{r}=0.50$ to 0.70$)$, low and negligible correlation $(\mathrm{r}<0.50)$ [25].

Table 4. Results of the inter-parameter correlation analysis.

\begin{tabular}{|c|c|c|c|c|c|c|}
\hline Corr. Index & $\overline{\mathrm{AA}}$ & EA & PAMH & PAPCE & SAMH & LASS \\
\hline $\mathrm{MPA}_{V \text {-mean }}$ & -0.537 & -0.421 & -0.754 & -0.866 & -0.708 & -0.829 \\
\hline $\mathrm{RPA}_{\mathrm{V} \text {-mean }}$ & -0.342 & -0.383 & -0.495 & -0.619 & -0.550 & -0.613 \\
\hline $\mathrm{LPA}_{\mathrm{V} \text {-mean }}$ & -0.537 & -0.163 & -0.723 & -0.743 & -0.682 & -0.817 \\
\hline RPSA $_{V \text {-mean }}$ & -0.220 & -0.529 & -0.761 & -0.725 & -0.816 & -0.819 \\
\hline LPSA $_{V \text {-mean }}$ & -0.342 & -0.565 & -0.827 & -0.785 & -0.892 & -0.925 \\
\hline RPSA-RPV $_{\Delta \mathrm{P} \text {-mean }}$ & -0.416 & -0.566 & -0.792 & -0.662 & -0.854 & -0.868 \\
\hline LPSA-LPV $_{\Delta \mathrm{P} \text {-mean }}$ & -0.390 & -0.526 & -0.851 & -0.742 & $\begin{array}{l}-0.917 \\
\end{array}$ & -0.915 \\
\hline
\end{tabular}

Red: Very strong correlation ( $r=0.90$ to 1.0); Yellow: Strong correlation $(r=0.70$ to 0.90$)$; Green: Moderate correlation $(\mathrm{r}=0.50$ to 0.70$)$; Blue: weak and negligible correlation $(\mathrm{r}<0.50)$. (MPA $\mathrm{M}_{\mathrm{V}-\mathrm{mean}}$ : Main pulmonary artery averaged velocity; $\mathrm{RPA}_{\mathrm{V} \text {-mean }}$ : Right pulmonary artery averaged velocity; $\mathrm{LPA}_{\mathrm{V} \text {-mean }}$ : Left pulmonary artery averaged velocity; $\mathrm{RPSA}_{\mathrm{V} \text {-mean }}$ : Right pulmonary small artery averaged velocity; $\mathrm{LPSA}_{\mathrm{V} \text {-mean }}$ : Left pulmonary small artery averaged velocity; RPSA-RPV $\mathrm{VP}_{\triangle \mathrm{P} \text {-mean }}$ : Right pulmonary arteriovenous averaged pressure gradient; LPSA-LPV $_{\triangle \mathrm{P} \text {-mean: }}$ Left pulmonary arteriovenous averaged pressure gradient).

\section{Results}

- CMR-Derived Hemodynamics: The time-averaged mean-velocities $\left(\mathrm{V}_{\text {mean }}\right)$ of the large and small pulmonary arteries were investigated in all the experimental groups. In addition, the right and left pulmonary arteriovenous pressure gradients (right: RPSA-RPV $V_{\triangle \mathrm{P} \text {-mean, left: LPSA-LPV }} \Delta \mathrm{P}$-mean $)$ were calculated (Table 1 ). The $\mathrm{V}_{\text {mean- }}$ related indices in the large pulmonary arteries were only capable of distinguishing between severe PAH and baseline $(p<0.05)$, while the $\mathrm{V}_{\text {mean }}$-related indices in the small pulmonary arteries were capable of differentiating not only between severe PAH and baseline, but also between the moderate and severe stages of the disease $(p<0.05)$. Notably, RPSA-RPV $\Delta$ P-mean and LPSA-LPV $\Delta$ P-mean could differentiate between baseline and severe PAH as well as the moderate and severe disease groups $(p<0.05)$, but with stronger statistical properties than $\mathrm{V}_{\text {mean, }}$ suggesting their suitability for the non-invasive diagnosis and follow-up of PAH. The qualitative assessments of the velocity-time curves in the pulmonary arteries indicated that the early and peak systolic velocities did not generally change in PAH. However, the late systolic velocities appeared more attenuated and flattened; i.e., velocity notches occurred (Figure 2, Figure 3 and Supplementary Material Video S1), which affected the $V_{\text {mean }}$ values. This 
phenomenon was more dominant in the small pulmonary arteries compared to the large ones.

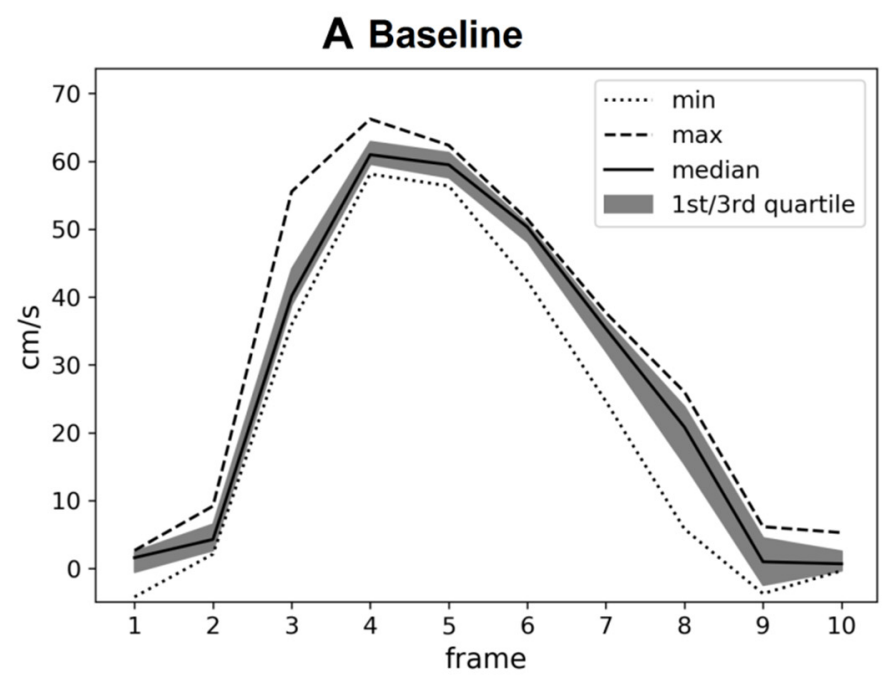

C Moderate PAH

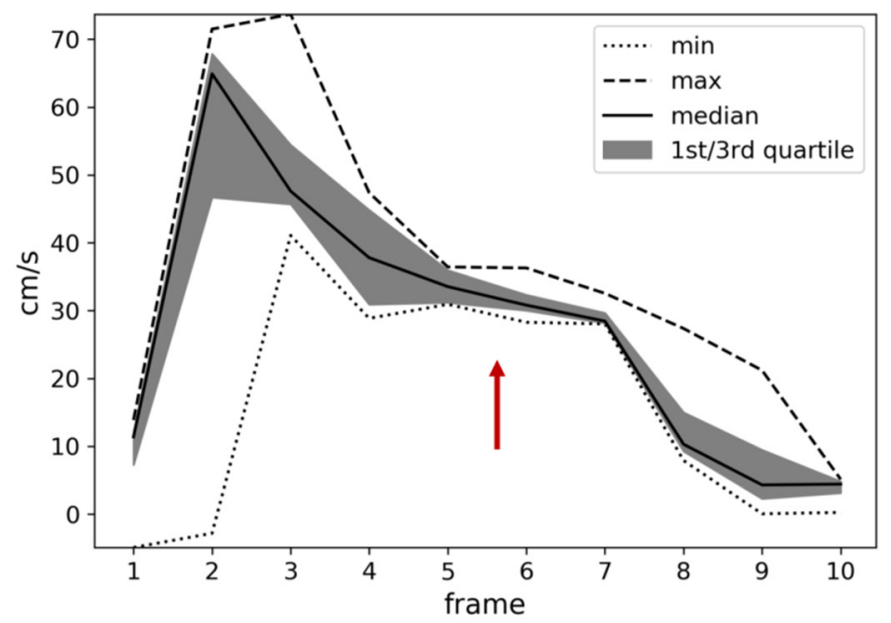

B Severe PAH

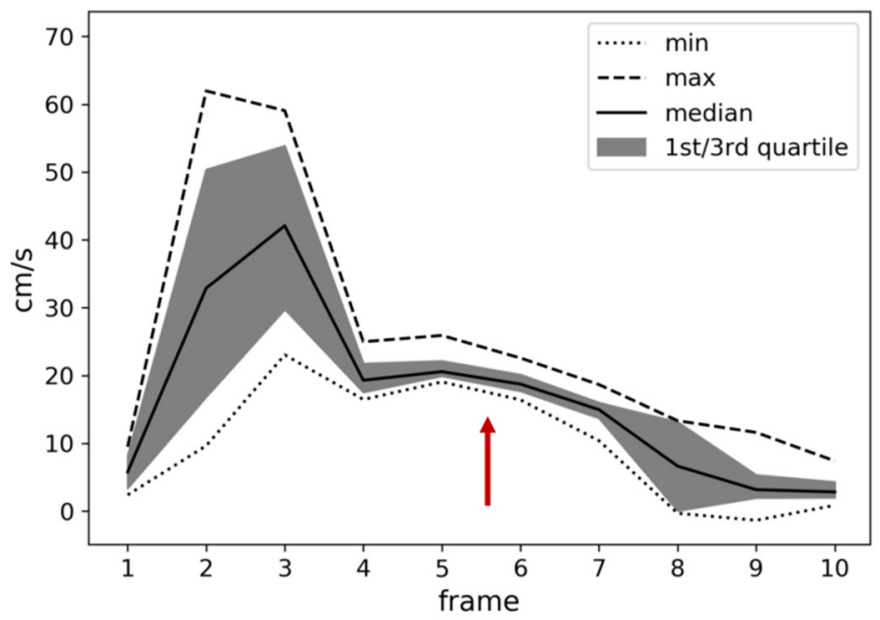

D Total Variation of All Groups

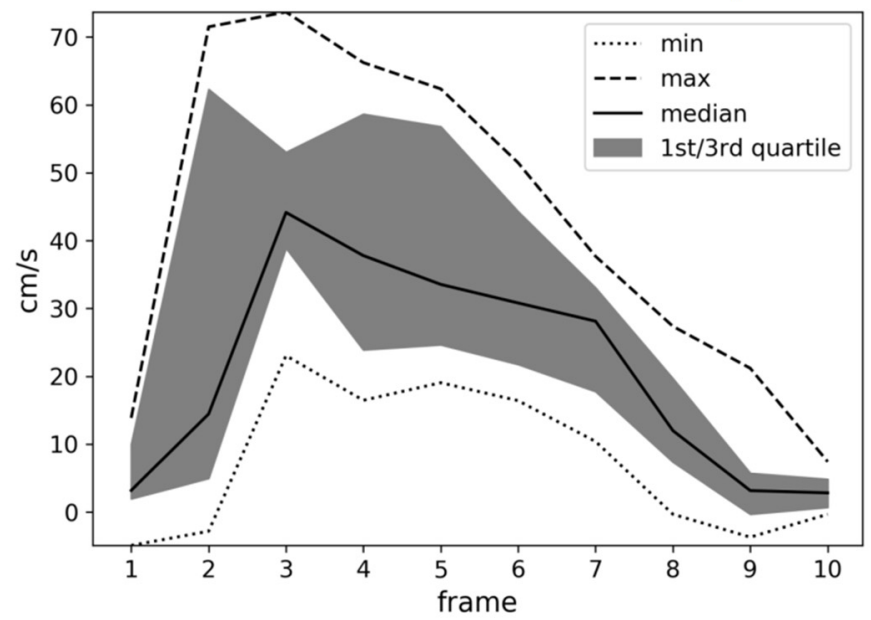

Figure 2. (A-C) Intra- and (D) inter-experimental group variability assessment of the velocity-time curve through a cross-section drawn in the distal portion of the main pulmonary artery, i.e., proximal to the pulmonary bifurcation. Notches are indicated by red arrows. Each cardiac frame corresponds to a temporal resolution of $10 \mathrm{~ms}$. 

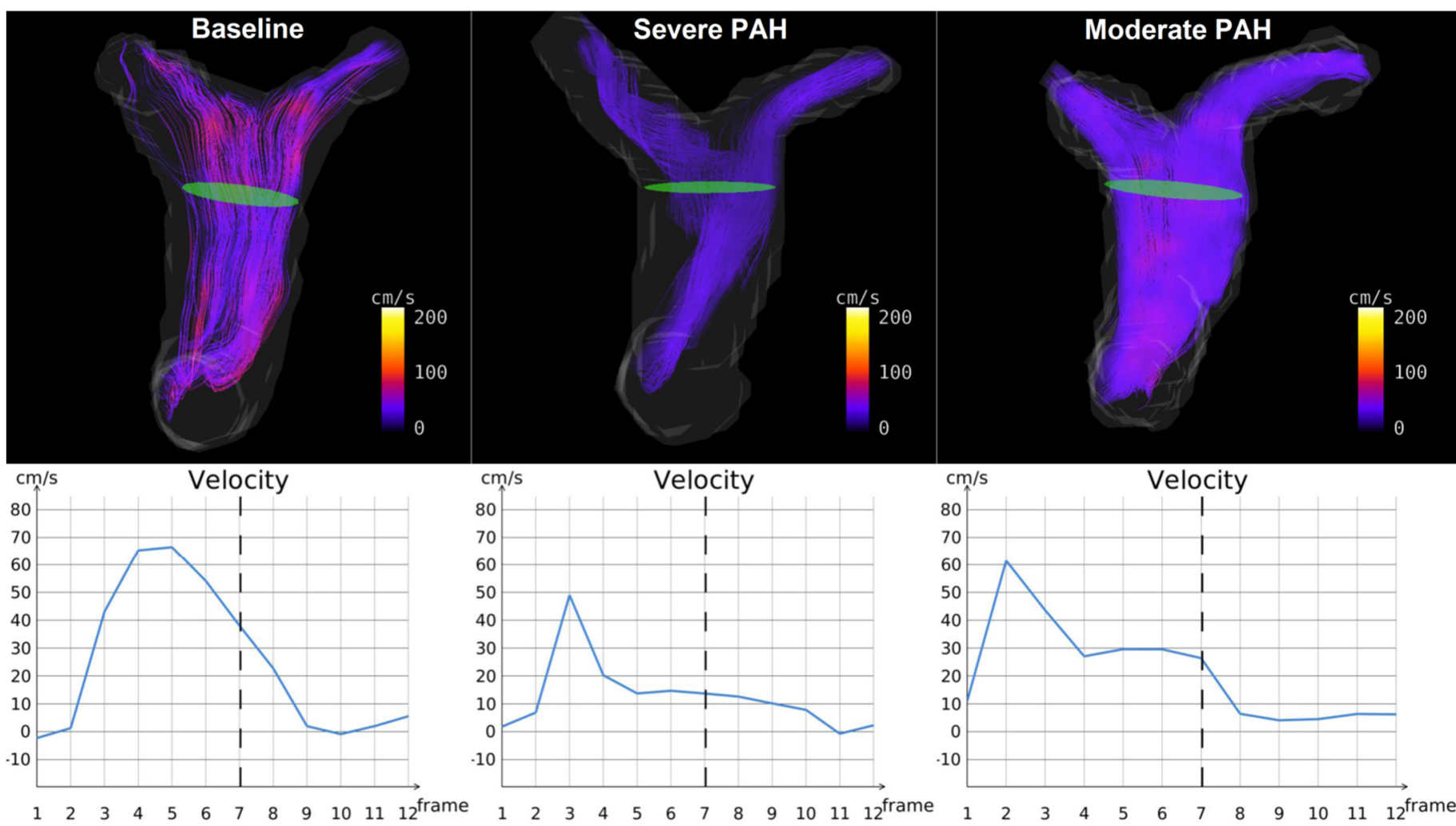

Figure 3. Representative streamline reconstructions of baseline, moderate, and severe pulmonary arterial hypertension in an animal model (top row) with corresponding velocity changes over time, averaged across the green cross-section in the distal MPA (bottom row). The vortical flow pattern formation and its effect on the corresponding velocity-time curve can be observed in this example in the green cross-section area.

- RVEF \& TAPSE: Due to the pitfalls and difficult procedure of RHC in rats, RVEF and TAPSE were investigated by CMR and TTE to prove all the animals were influenced by PAH. RVEF could reliably differentiate all the experimental groups from each other $(p<0.05)$, i.e., it was largely reduced in severe PAH and preserved in the moderate stage (Table 2). TAPSE could also differentiate well between baseline and severe PAH as well as between the severe and moderate disease groups $(p<0.05)($ Table 2$)$ (Figure 4). The RVEF and TAPSE results proved the effectiveness of the experimental design. 

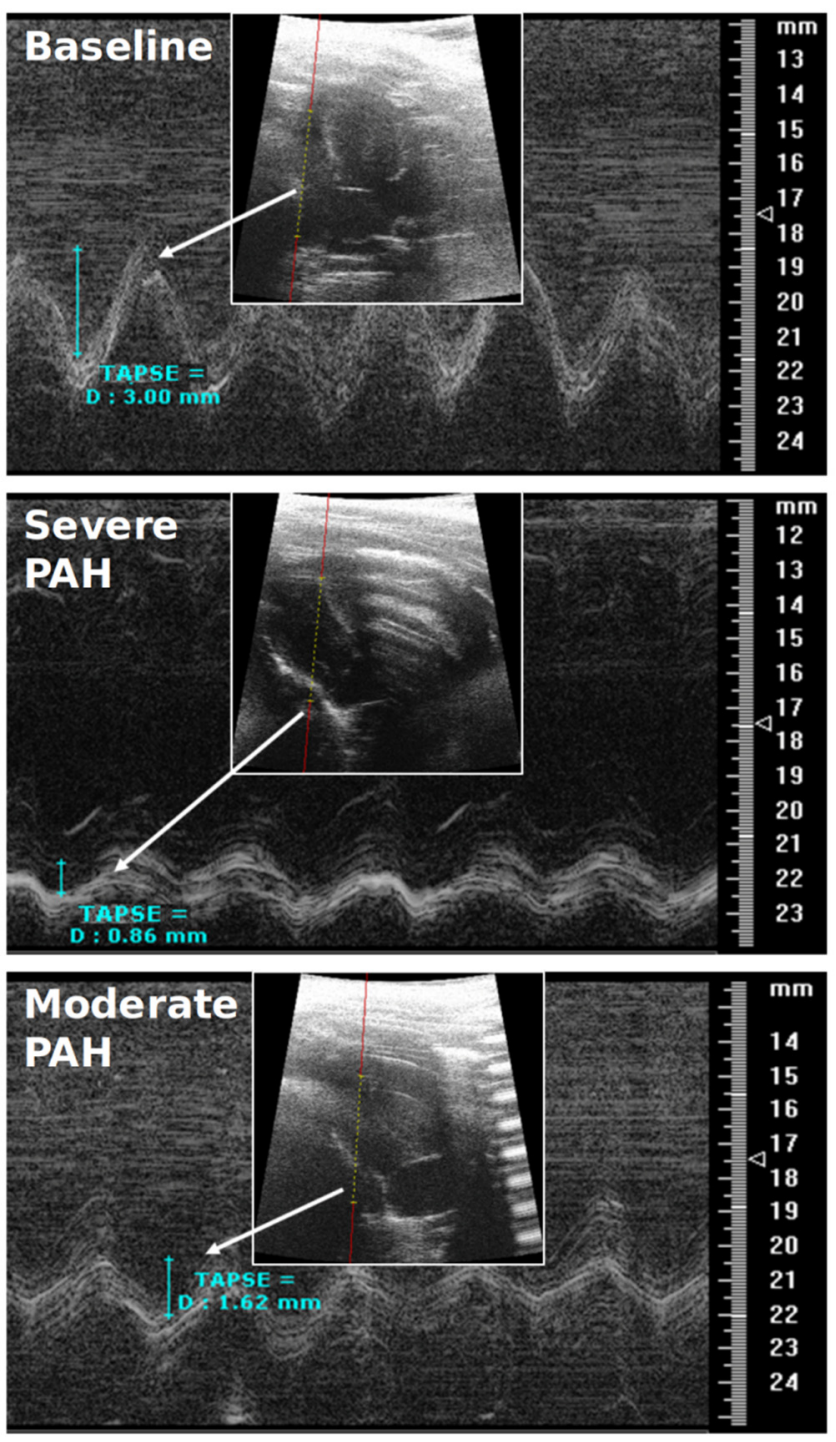

Figure 4. Representative transthoracic echocardiography (TTE) images showing the tricuspid annular plane systolic excursion (TAPSE) in baseline, moderate, and severe pulmonary arterial hypertension.

- Histological Assessment of Lung Tissue: In general, the animals with severe PAH demonstrated the highest individual scores in all the histological criteria except for emphysema among all the experimental groups. Therefore, severe PAH showed extensive signs of histological damage compared to baseline through significantly higher sumscores $(p<0.05)$ (Figure 5). In moderate PAH, the sum-score was significantly lower compared to the severe group but still higher than the baseline $(p<0.05)$, reflecting a partial improvement in histological damage. With respect to the individual indices, moderate PAH showed a significant decrease for atelectasis and media hypertrophy in both the peribronchial and small pulmonary arteries compared to the animals at the severe stage of the disease $(p<0.05)$. However, the media hypertrophy of both arteries was still increased in severe and moderate PAH compared to baseline $(p<0.05)$ (Figure 5). 
A Baseline

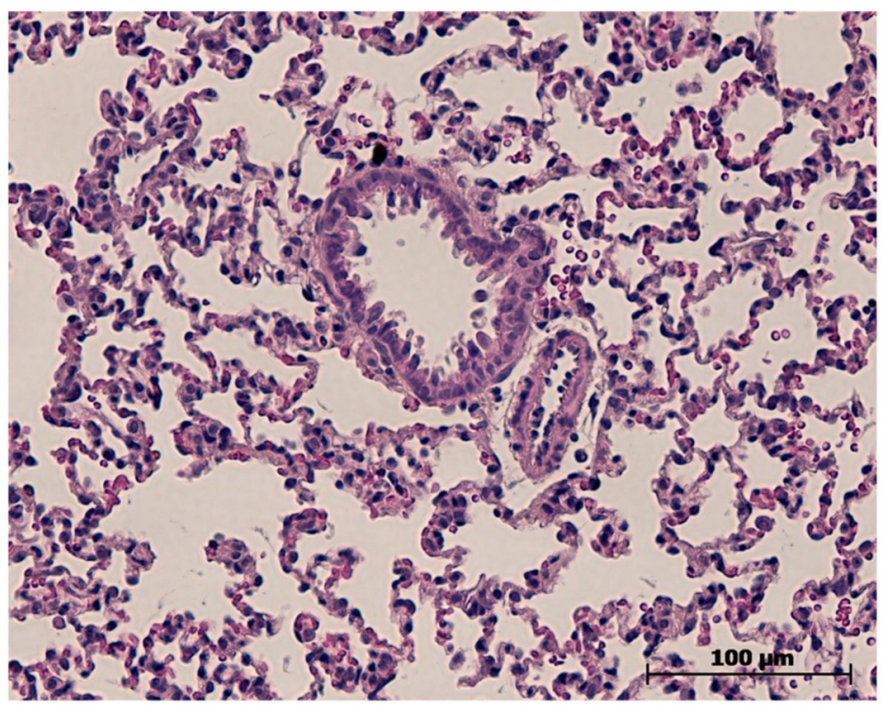

C Moderate PAH
B Severe PAH

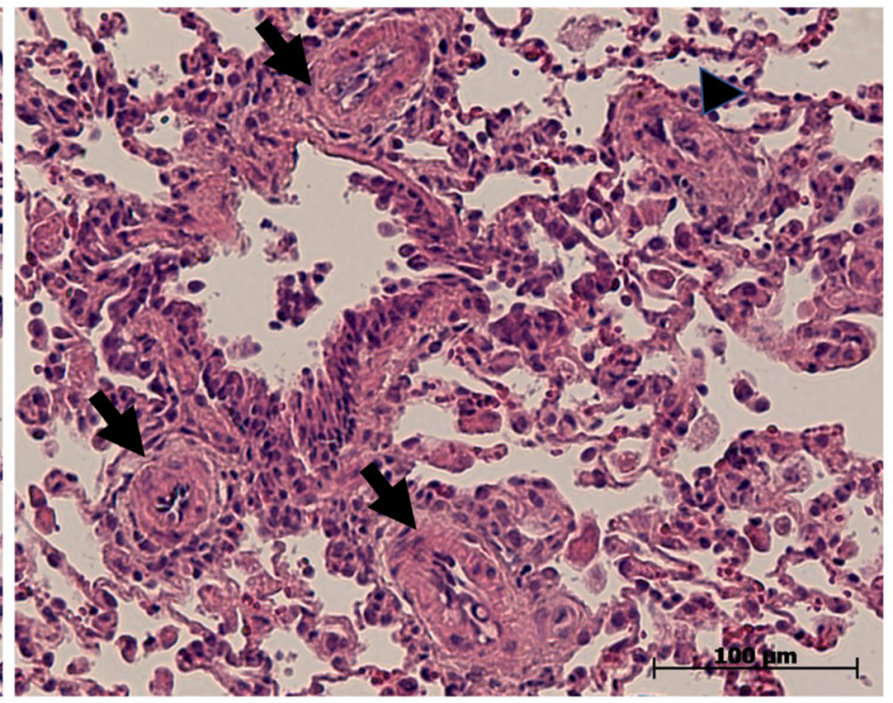

D Semiquantitative Assessment
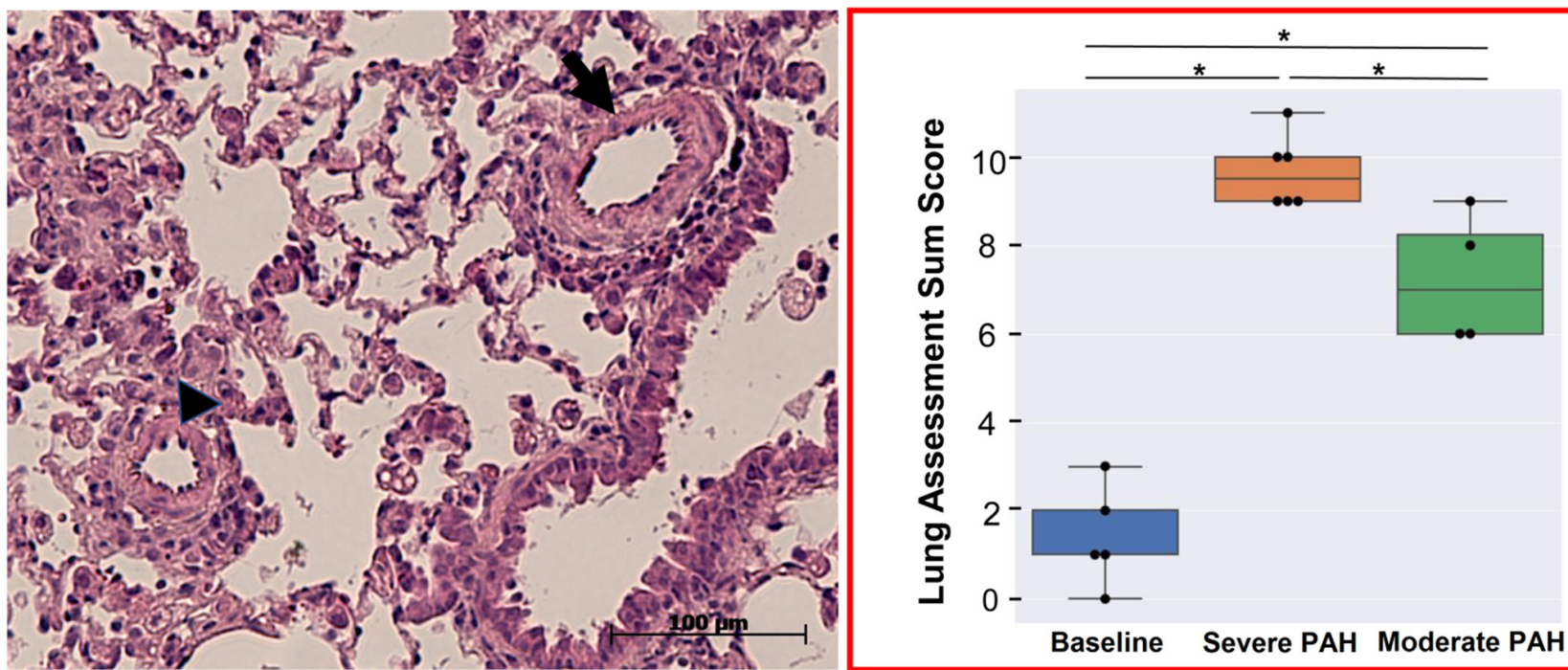

Figure 5. Representative images of histological changes in the lung tissue of baseline, moderate, and severe pulmonary arterial hypertension $(\mathrm{PAH})$ with a focus on media hypertrophy of peribronchial (black arrows) and small arteries (black arrowheads) at a $20 \times$ magnification, as well as a graphical comparison between histological sum-score changes between all three experimental groups ( ${ }^{*}$ indicates statistical significance, with $p<0.05$ ).

- Correlation analysis between 4D-flow parameters and histopathology: In general, the correlation analysis between the $\mathrm{V}_{\text {mean-related indices and the histological results }}$ showed moderate-to-strong correlation coefficient values, while the correlation analysis between the right/left pulmonary arteriovenous pressure gradients and histological results indicated a strong-to-very-strong associativity (Table 4).

\section{Discussion and Conclusions}

The limitations of RHC in the diagnosis of PAH in combination with its invasiveness prompted us to investigate the potential role of new hemodynamic indices derived from 4D-flow to diagnose PAH. In current research, CMR is used to assess the response of $\mathrm{PH}$ to surgical or medical treatments primarily through conventional indices (e.g., right ventricular functions, right ventricular mass, main pulmonary velocities, etc.) [16]. In addition, the hemodynamic changes in the large pulmonary arteries under $\mathrm{PH}$ have been 
extensively assessed in previous studies and are well summarized by Reiter U. et al. [19]. In PAH, we observed the same findings as in the previous clinical studies [21,22,26-31], indicating that we reached the same level of accuracy. In a recent study by Cerne et al. [32], the $V_{\text {mean }}$ of the main, right and left pulmonary arteries showed considerably lower values in PAH in comparison to the normal population. However, in the current study, we hypothesized that the small pulmonary arteries were more suitable regions than large vessels for the evaluation of hemodynamic changes due to their proximity to the site of media hypertrophy. In contrast to [32], we chose histology as the reference standard instead of RHC to directly evaluate the lung tissue changes in PAH, since hemodynamics alter secondary to histological changes. In addition, we minimized the time gaps between all the diagnostic procedures and kept all the study conditions constant throughout the experiment to mitigate any possible confounding factors arising in between. In our results, the $\mathrm{V}_{\text {mean }}$ of the small pulmonary arteries differentiated between the baseline, moderate, and severe PAH groups more sensitively than the $\mathrm{V}_{\text {mean }}$ of the large pulmonary arteries, suggesting its superiority as a hemodynamic index in diagnosing PAH.

In addition, the pronounced changes in the $\mathrm{V}_{\text {mean }}$ of the small pulmonary arteries in severe PAH reflected the hampered blood flow within the pulmonary circulation as a result of vascular resistance, which led to blood flow pattern changes and vortex ring formation in the large pulmonary arteries (Figures 2 and 3, Supplementary Material Video S1). Vortex rings influenced the shape of the velocity-time curves and could adequately explain the formation of the velocity notches during the late systole, which was also described in $[19,33]$. Figure 3 and Supplementary Material Video S1 highlight an exemplary case, illustrating the effects of vortex ring formation on the velocity-time curves in the distal portion of the main pulmonary artery. The study by Reiter G. et al. [34] also reported a correlation between vortex ring formation and mean pulmonary artery pressure changes in $\mathrm{PH}$, which supports our observations.

In addition to the evaluation of the $V_{\text {mean }}$ in the small pulmonary arteries, we introduced the time-averaged pulmonary arteriovenous pressure gradient as a new diagnostic index. We modeled the pulmonary circulation in the lungs as a simple water stopcock system with one inlet and one outlet (i.e., the vascular stiffness of the lung was modeled as the stopcock, the pulmonary artery was the inlet, and the pulmonary vein was the outlet) and used the modified Bernoulli's equation to roughly measure the associated pressure gradient caused by the stopcock stiffness, which was equivalent to the severity of media hypertrophy in our model of PAH. This study showed that the pulmonary arteriovenous pressure gradient was one of the most sensitive indices reflecting the severity of PAH. The correlation between this novel parameter and the lung assessment sum-score in histology was found to be very strong (more than $90 \%$ ), which is superior to all the previously introduced imaging biomarkers summarized in [19].

As stated in the Materials and Methods section, the target regions of this study were the small pulmonary arteries. To meet the necessary spatial properties of such small geometries, the resolution and the measurement noise had to be increased; however, the use of the $4 \mathrm{D}$-flow stack-of-stars velocity mapping technique instead of the $4 \mathrm{D}$-flow Cartesian technique (which is the most frequently used sequence in preclinical CMR) could compensate for the loss of velocity-to-noise ratio and could decrease the measurement bias in these small vessels.

The major pitfall of this study was the high standard deviations in different hemodynamic indices observed in moderate PAH. A reasonable explanation could be that the response to medication was subjective and could vary among individual animals, which might have led to large standard deviations in the results of this group. One suitable remedy for this problem could be an increase in the sample size to allow more accurate statistics. In addition, we recommend a more comprehensive longitudinal study with multiple CMR scans on individual animals at multiple time points during the disease progression, instead of one single time point. Despite all these constraints, we are convinced that the results of the time-averaged pulmonary arteriovenous pressure gradient and the velocity values in 
the small pulmonary arteries can serve as potentially sensitive biomarkers in the diagnosis and follow-up of PAH.

Supplementary Materials: The following supporting information can be downloaded at: https: / / www.mdpi.com/article/10.3390/diagnostics12010058/s1. Video S1: Qualitative assessment of the velocity-time curves in the main pulmonary artery. Due to high vascular resistance in severe pulmonary arterial hypertension (PAH in diseased animals), vortex rings are generated in the large pulmonary arteries producing velocity notches in the corresponding velocity-time curves. This effect was not noticed in either the baseline (normal) or in the moderate PAH (treated) animals.

Author Contributions: Conceptualization: A.N., M.F. and V.H.; methodology (CMR): A.N., V.H., M.K., K.-H.H. and J.R.R.; methodology (Histology): K.G. and M.F.; methodology (TTE): M.F. and A.S.; methodology (Animal model): M.F., K.G. and C.J.; Software: A.N., S.M., M.K. and V.H.; Validation: K.G., M.F., C.J., A.N. and V.H.; Formal analysis: A.N., M.F. and V.H.; Investigation: A.N., S.L., K.G., M.K., K.-H.H., A.S., C.J., S.M., P.C.S., L.L., J.R.R., V.H. and M.F.; Resources: K.-H.H., J.R.R., M.F. and V.H.; Writing—original draft preparation: A.N. and V.H.; Writing—review and editing: J.R.R., M.F., P.C.S., A.N. and V.H.; Visualization: A.N., V.H., S.L., L.L. and S.M.; Supervision: V.H. and M.F.; Project administration: M.F. and V.H.; Funding acquisition: V.H. and M.F. All authors have read and agreed to the published version of the manuscript.

Funding: This study was supported by the Federal Ministry of Education and Research (BMBF), Germany, FKZ 01EO1502 and the publication was funded by the Open Access Publication Fund of the University of Bonn.

Institutional Review Board Statement: All the experiments were conducted in accordance with the National Institute of Health Guidelines for the Care and Use of Laboratory Animals (8th edition) and the European Community Council Directive for the Care and Use of Laboratory Animals of 22 September 2010 (2010/63/EU). The study protocol was approved by the competent State Office of Food Safety and Consumer Protection (TLLV, Bad Langensalza, Germany; local registration number: UKJ-17-003).

Informed Consent Statement: Not applicable.

Data Availability Statement: The corresponding data related to this study are available upon request.

Acknowledgments: The authors thank Moritz Wildgruber for valuable discussions on the project. The macitentan was provided by Actelion Pharmaceuticals Ltd., under a material transfer agreement. The company was not involved in the study design, conduct, or interpretation. Parts of this work will be used in the doctoral thesis of Ali Nahardani.

Conflicts of Interest: The authors declare no conflict of interest.

\section{References}

1. Galiè, N.; Humbert, M.; Vachiery, J.L.; Gibbs, S.; Lang, I.; Torbicki, A.; Simonneau, G.; Peacock, A.; Vonk Noordegraaf, A.; Beghetti, M.; et al. 2015 ESC/ERS Guidelines for the diagnosis and treatment of pulmonary hypertension: The Joint Task Force for the Diagnosis and Treatment of Pulmonary Hypertension of the European Society of Cardiology (ESC) and the European Respiratory Society (ERS): Endorsed by: Association for European Paediatric and Congenital Cardiology (AEPC), International Society for Heart and Lung Transplantation (ISHLT). Eur. Heart J. 2016, 37, 67-119. [CrossRef] [PubMed]

2. Hoeper, M.M.; Bogaard, H.J.; Condliffe, R.; Frantz, R.; Khanna, D.; Kurzyna, M.; Langleben, D.; Manes, A.; Satoh, T.; Torres, F.; et al. Definitions and diagnosis of pulmonary hypertension. J. Am. Coll. Cardiol. 2013, 62, D42-D50. [CrossRef] [PubMed]

3. Rich, J.D.; Rich, S. Clinical diagnosis of pulmonary hypertension. Circulation 2014, 130, 1820-1830. [CrossRef] [PubMed]

4. Speich, R. Diagnosing pulmonary hypertension: Is there a revival of the electrocardiogram? Eur. Respir. J. 2011, 37, 994-996. [CrossRef] [PubMed]

5. Barbosa, E.J.M.; Gupta, N.K.; Torigian, D.A.; Gefter, W.B. Current Role of Imaging in the Diagnosis and Management of Pulmonary Hypertension. Am. J. Roentgenol. 2012, 198, 1320-1331. [CrossRef]

6. Grignola, J.C. Hemodynamic assessment of pulmonary hypertension. World J. Cardiol. 2011, 3, 10-17. [CrossRef]

7. Rosenkranz, S.; Preston, I.R. Right heart catheterisation: Best practice and pitfalls in pulmonary hypertension. Eur. Respir. Rev. Off. J. Eur. Respir. Soc. 2015, 24, 642-652. [CrossRef]

8. Mathier, M.A. The Nuts and Bolts of Interpreting Hemodynamics in Pulmonary Hypertension Associated With Diastolic Heart Failure. Adv. Pulm. Hypertens. 2011, 10, 33-40. [CrossRef] 
9. Hoeper, M.M.; Lee, S.H.; Voswinckel, R.; Palazzini, M.; Jais, X.; Marinelli, A.; Barst, R.J.; Ghofrani, H.A.; Jing, Z.C.; Opitz, C.; et al. Complications of right heart catheterization procedures in patients with pulmonary hypertension in experienced centers. J. Am. Coll. Cardiol. 2006, 48, 2546-2552. [CrossRef]

10. Rudski, L.G.; Lai, W.W.; Afilalo, J.; Hua, L.; Handschumacher, M.D.; Chandrasekaran, K.; Solomon, S.D.; Louie, E.K.; Schiller, N.B. Guidelines for the echocardiographic assessment of the right heart in adults: A report from the American Society of Echocardiography endorsed by the European Association of Echocardiography, a registered branch of the European Society of Cardiology, and the Canadian Society of Echocardiography. J. Am. Soc. Echocardiogr. Off. Publ. Am. Soc. Echocardiogr. 2010, 23, 685-713. [CrossRef]

11. Ascha, M.; Renapurkar, R.D.; Tonelli, A.R. A review of imaging modalities in pulmonary hypertension. Ann. Thorac. Med. 2017, 12, 61-73. [CrossRef]

12. Meng, J.J.; Zhang, L.J.; Wang, Q.; Fang, W.; Dai, H.J.; Yan, J.; Wang, T.; Yao, Z.M.; He, J.; Li, M.; et al. A comparison of ventilation/perfusion single photon emission CT and CT pulmonary angiography for diagnosis of pulmonary embolism. Chin. J. Tuberc. Respir. Dis. 2013, 36, 177-181. (In Chinese)

13. Tunariu, N.; Gibbs, S.J.; Win, Z.; Gin-Sing, W.; Graham, A.; Gishen, P.; Al-Nahhas, A. Ventilation-perfusion scintigraphy is more sensitive than multidetector CTPA in detecting chronic thromboembolic pulmonary disease as a treatable cause of pulmonary hypertension. J. Nucl. Med. Off. Publ. Soc. Nucl. Med. 2007, 48, 680-684. [CrossRef]

14. Moledina, S.; Pandya, B.; Bartsota, M.; Mortensen, K.H.; McMillan, M.; Quyam, S.; Taylor, A.M.; Haworth, S.G.; Schulze-Neick, I.; Muthurangu, V. Prognostic significance of cardiac magnetic resonance imaging in children with pulmonary hypertension. Circ. Cardiovasc. Imaging 2013, 6, 407-414. [CrossRef]

15. Bradlow, W.M.; Gibbs, J.S.; Mohiaddin, R.H. Cardiovascular magnetic resonance in pulmonary hypertension. J. Cardiovasc. Magn. Reson. Off. J. Soc. Cardiovasc. Magn. Reson. 2012, 14, 6. [CrossRef] [PubMed]

16. Peacock, A.J.; Vonk Noordegraaf, A. Cardiac magnetic resonance imaging in pulmonary arterial hypertension. Eur. Respir. Rev. Off. J. Eur. Respir. Soc. 2013, 22, 526-534. [CrossRef]

17. Freed, B.H.; Collins, J.D.; François, C.J.; Barker, A.J.; Cuttica, M.J.; Chesler, N.C.; Markl, M.; Shah, S.J. MR and CT Imaging for the Evaluation of Pulmonary Hypertension. JACC Cardiovasc. Imaging 2016, 9, 715-732. [CrossRef] [PubMed]

18. Dyverfeldt, P.; Bissell, M.; Barker, A.J.; Bolger, A.F.; Carlhäll, C.J.; Ebbers, T.; Francios, C.J.; Frydrychowicz, A.; Geiger, J.; Giese, D.; et al. 4D flow cardiovascular magnetic resonance consensus statement. J. Cardiovasc. Magn. Reson. Off. J. Soc. Cardiovasc. Magn. Reson. 2015, 17, 72. [CrossRef]

19. Reiter, U.; Reiter, G.; Fuchsjäger, M. MR phase-contrast imaging in pulmonary hypertension. Br. J. Radiol. 2016, 89, 20150995. [CrossRef]

20. Roldán-Alzate, A.; Frydrychowicz, A.; Johnson, K.M.; Kellihan, H.; Chesler, N.C.; Wieben, O.; François, C.J. Non-invasive assessment of cardiac function and pulmonary vascular resistance in an canine model of acute thromboembolic pulmonary hypertension using 4D flow cardiovascular magnetic resonance. J. Cardiovasc. Magn. Reson. Off. J. Soc. Cardiovasc. Magn. Reson. 2014, 16, 23. [CrossRef] [PubMed]

21. García-Alvarez, A.; Fernández-Friera, L.; Mirelis, J.G.; Sawit, S.; Nair, A.; Kallman, J.; Fuster, V.; Sanz, J. Non-invasive estimation of pulmonary vascular resistance with cardiac magnetic resonance. Eur. Heart J. 2011, 32, 2438-2445. [CrossRef]

22. Sanz, J.; Kuschnir, P.; Rius, T.; Salguero, R.; Sulica, R.; Einstein, A.J.; Dellegrottaglie, S.; Fuster, V.; Rajagopalan, S.; Poon, M Pulmonary arterial hypertension: Noninvasive detection with phase-contrast MR imaging. Radiology 2007, 243, 70-79. [CrossRef]

23. Franz, M.; Grün, K.; Betge, S.; Rohm, I.; Ndongson-Dongmo, B.; Bauer, R.; Schulze, P.C.; Lichtenauer, M.; Petersen, I.; Neri, D.; et al. Lung tissue remodelling in MCT-induced pulmonary hypertension: A proposal for a novel scoring system and changes in extracellular matrix and fibrosis associated gene expression. Oncotarget 2016, 7, 81241-81254. [CrossRef] [PubMed]

24. Rohm, I.; Grün, K.; Müller, L.M.; Bäz, L.; Förster, M.; Schrepper, A.; Kretzschmar, D.; Pistulli, R.; Yilmaz, A.; Bauer, R.; et al. Cellular inflammation in pulmonary hypertension: Detailed analysis of lung and right ventricular tissue, circulating immune cells and effects of a dual endothelin receptor antagonist. Clin. Hemorheol. Microcirc. 2019, 73, 497-522. [CrossRef] [PubMed]

25. Mukaka, M.M. Statistics corner: A guide to appropriate use of correlation coefficient in medical research. Malawi Med. J. 2012, 24, 69-71.

26. Guo, X.; Liu, M.; Ma, Z.; Wang, S.; Yang, Y.; Zhai, Z.; Wang, C.; Zhai, R. Flow characteristics of the proximal pulmonary arteries and vena cava in patients with chronic thromboembolic pulmonary hypertension: Correlation between $3.0 \mathrm{~T}$ phase-contrast MRI and right heart catheterization. Diagn. Interv. Radiol. 2014, 20, 414-420. [CrossRef] [PubMed]

27. Helderman, F.; Mauritz, G.J.; Andringa, K.E.; Vonk-Noordegraaf, A.; Marcus, J.T. Early onset of retrograde flow in the main pulmonary artery is a characteristic of pulmonary arterial hypertension. J. Magn. Reson. Imaging JMRI 2011, 33, 1362-1368. [CrossRef]

28. Ley, S.; Fink, C.; Risse, F.; Ehlken, N.; Fischer, C.; Ley-Zaporozhan, J.; Kauczor, H.U.; Klose, H.; Gruenig, E. Magnetic resonance imaging to assess the effect of exercise training on pulmonary perfusion and blood flow in patients with pulmonary hypertension. Eur. Radiol. 2013, 23, 324-331. [CrossRef]

29. Moral, S.; Fernández-Friera, L.; Stevens, G.; Guzman, G.; García-Alvarez, A.; Nair, A.; Evangelista, A.; Fuster, V.; Garcia, M.J.; Sanz, J. New index $\alpha$ improves detection of pulmonary hypertension in comparison with other cardiac magnetic resonance indices. Int. J. Cardiol. 2012, 161, 25-30. [CrossRef] 
30. Swift, A.J.; Rajaram, S.; Condliffe, R.; Capener, D.; Hurdman, J.; Elliot, C.A.; Wild, J.M.; Kiely, D.G. Diagnostic accuracy of cardiovascular magnetic resonance imaging of right ventricular morphology and function in the assessment of suspected pulmonary hypertension results from the ASPIRE registry. J. Cardiovasc. Magn. Reson. Off. J. Soc. Cardiovasc. Magn. Reson. 2012, 14, 40. [CrossRef]

31. Truong, U.; Fonseca, B.; Dunning, J.; Burgett, S.; Lanning, C.; Ivy, D.D.; Shandas, R.; Hunter, K.; Barker, A.J. Wall shear stress measured by phase contrast cardiovascular magnetic resonance in children and adolescents with pulmonary arterial hypertension. J. Cardiovasc. Magn. Reson. Off. J. Soc. Cardiovasc. Magn. Reson. 2013, 15, 81. [CrossRef] [PubMed]

32. Cerne, J.W.; Pathrose, A.; Gordon, D.Z.; Sarnari, R.; Veer, M.; Blaisdell, J.; Allen, B.D.; Avery, R.; Markl, M.; Ragin, A.; et al. Evaluation of Pulmonary Hypertension Using 4D Flow MRI. J. Magn. Reson. Imaging 2021. [CrossRef] [PubMed]

33. Kjellström, B.; Lindholm, A.; Ostenfeld, E. Cardiac Magnetic Resonance Imaging in Pulmonary Arterial Hypertension: Ready for Clinical Practice and Guidelines? Curr. Heart Fail. Rep. 2020, 17, 181-191. [CrossRef]

34. Reiter, G.; Reiter, U.; Kovacs, G.; Olschewski, H.; Fuchsjäger, M. Blood flow vortices along the main pulmonary artery measured with MR imaging for diagnosis of pulmonary hypertension. Radiology 2015, 275, 71-79. [CrossRef] [PubMed] 